\title{
Out-of-Plane Nanoscale Reorganization of Lipid Molecules and Nanoparticles Revealed by Plasmonic Spectroscopy
}

\author{
Matthew R. Cheetham, Jack Griffiths, Bart de Nijs, George R. Heath, Stephen D. Evans, \\ Jeremy J. Baumberg,* and Rohit Chikkaraddy*
}

Cite This: J. Phys. Chem. Lett. 2020, 11, 2875-2882

Read Online

ABSTRACT: Lipid bilayers assembled on solid substrates have been extensively studied with singlemolecule resolution as the constituent molecules diffuse in $2 \mathrm{D}$; however, the out-of-plane motion is typically ignored. Here we present the subnanometer out-of-plane diffusion of nanoparticles attached to hybrid lipid bilayers (HBLs) assembled on metal surfaces. The nanoscale cavity formed between the Au nanoparticle and Au film provides strongly enhanced optical fields capable of locally probing HBLs assembled in the gaps. This allows us to spectroscopically resolve the nanoparticles assembled on bilayers, near edges, and in membrane defects, showing the strong influence of charged lipid rafts. Nanoparticles sitting on the edges of the HBL are observed to flip onto and off of the bilayer, with flip energies of $\sim 10 \mathrm{meV}$ showing how thermal energies dynamically modify lipid arrangements around a nanoparticle. We further resolve the movement of individual lipid molecules by doping the HBL with low concentrations of Texas Red (TxR) dye-labeled lipids.

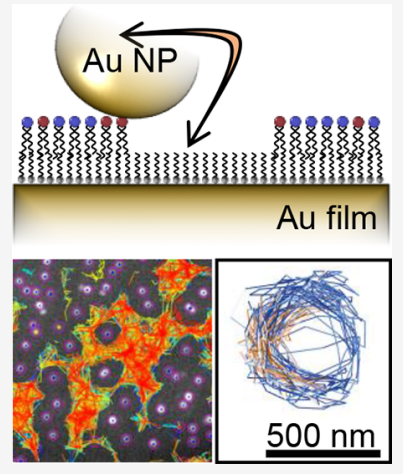

$\mathrm{L}$ ipid membranes are of vital importance to the function and survival of cells. Many of the processes in these membranes are understood only on a qualitative level, largely due to the small length scales and fast time scales involved. These systems are intrinsically fragile and environmentspecific, making them difficult to probe. The development of nanoscopic optical tools which minimally interfere with membrane function is thus in high demand. ${ }^{1,2}$ Metal nanoparticles (NPs) are used to track and image the diffusion of molecules and proteins within cell membranes, but the role of these NPs in modifying membrane dynamics remains unclear. ${ }^{3}$ NPs either rupture through the cell membrane furnishing their use as therapeutic agents ${ }^{4}$ and inducing cytotoxicity 5,6 or rest on the membrane providing imaging capabilities. These contradictory behaviors are linked to the different chemical and physical properties of NPs within membranes. $^{7-9}$

The complexity of lipid membrane diffusion has led to the "fluid mosaic" model ${ }^{10}$ being replaced with the "picket fence" model, ${ }^{11}$ accounting for the very inhomogeneous nature of cell membranes with a great deal of compartmentalization and scaffolding. This gives rise to anomalous diffusion within the bilayer, ${ }^{17,19}$ which cells are believed to control in order to alter reaction pathways. Here we use hybrid lipid bilayers (HBLs) as model systems and study their interaction with AuNPs to develop tools to better understand the nanoscale inhomogeneity of HBLs ${ }^{12,13}$ and the complex diffusion of NPs on such surfaces. $^{14,15}$

Advanced techniques to track the nanoscale diffusion of lipids within a membrane currently rely on tagging lipids with dyes $^{16-18}$ or nanoparticles ${ }^{13,15,19-22}$ and imaging using fluorescence or interferometric scattering, respectively. These show the in-plane (2D) diffusion of lipids with high spatial accuracy and time resolution but ignore nanoscale out-of-plane motion due to insensitivity to displacements in this dimension of $<10 \mathrm{~nm}$. Here we present the nanoscale out-of-plane motion of lipid molecules in supported bilayers and their reorganization on interaction with metal NPs.

Nanostructures made of noble metals, such as the AuNPs used here, strongly confine light below the diffraction limit with minimal perturbation to the local environment. Their strong optical signatures make them ideal for probing cell membranes. Optically excited collective oscillations of free electrons ("plasmons") on the metal confine optical fields tightly to a nanostructure. ${ }^{23,24}$ This localized optical field enhances light-matter interactions, allowing single-molecule sensitivity using Purcell-enhanced fluorescence (PL), surfaceenhanced Raman scattering (SERS), or absorption., ${ }^{1,25,26}$ However, this enhancement requires the assembly of metal nanostructures with narrow gaps or rod-like structures with large aspect ratios.

Here we construct nanoparticle-on-mirror (NPoM) cavities in which hybrid lipid bilayers (HBLs) are assembled between a

Received: January 17, 2020

Accepted: March 19, 2020

Published: March 19, 2020 

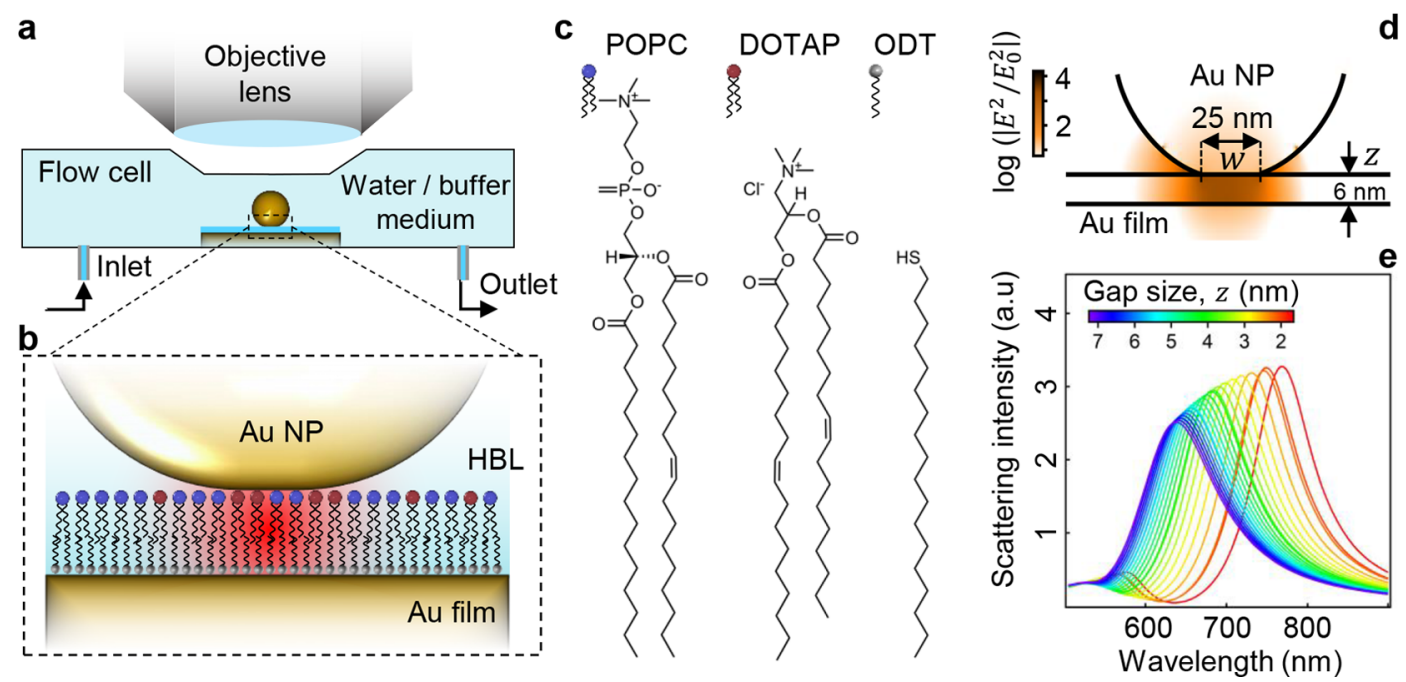

Figure 1. Experimental setup. (a) Schematic of the flow cell showing a sealed NPoM sample underneath a $100 \times$ long-working-distance 0.8 NA objective. (b) Enlarged schematic of NPoM, showing a hybrid bilayer in a plasmonic cavity formed between the AuNP and Au film. (c) Chemical structures of the bilayer constituents. (d) Three-dimensional FDTD simulation showing the spatial distribution of enhanced plasmonic intensity. (e) Simulated scattering spectra from NPoMs with various gaps, used to calibrate the dark-field resonance peak vs gap size.
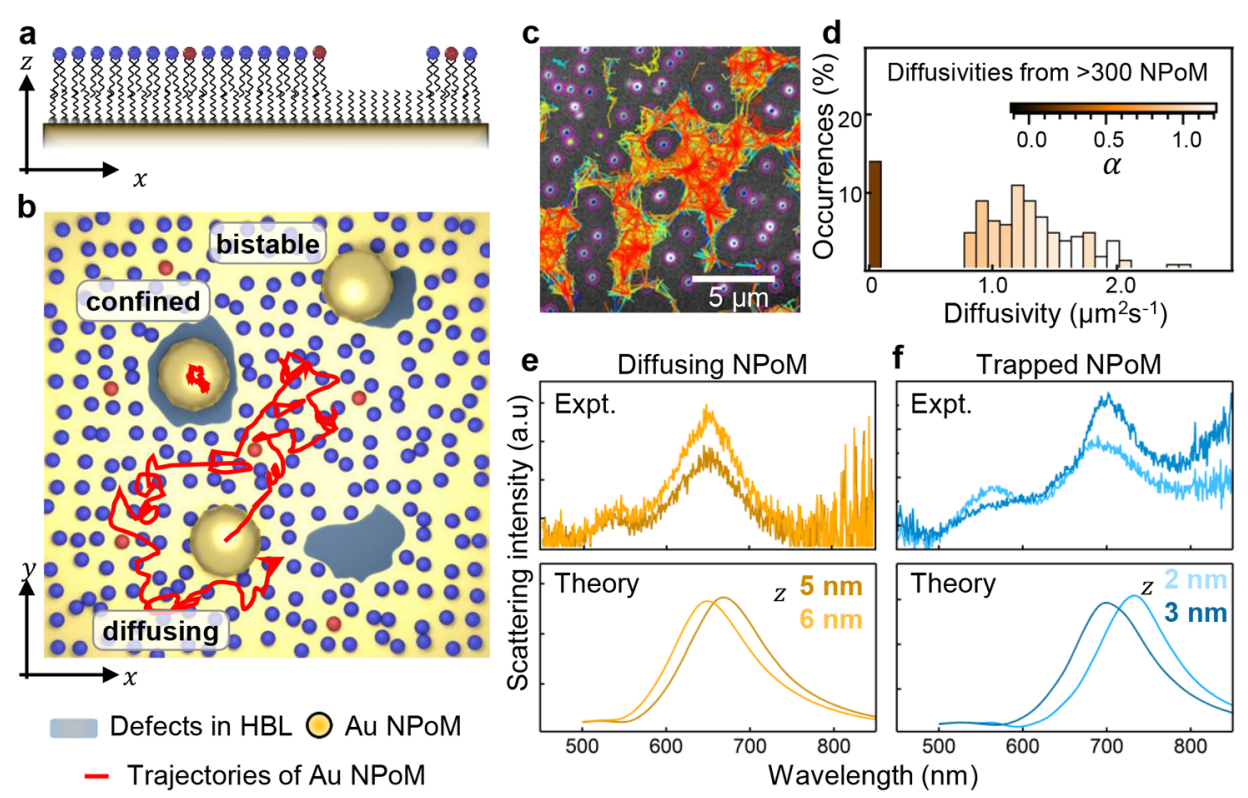

Figure 2. (a) Side view of a hybrid bilayer with a defect. (b) Top-down schematic with defects and AuNPs, together with representative diffusion trajectories (red tracks). (c) Example image from analyzed dark-field video showing AuNPs on the bilayer. Stuck AuNPs exhibit a confined diffusion (purple circles). The trajectories of other AuNPs are tracked with the trajectory color indicating their diffusivities and (d) showing a histogram of diffusivities obtained from the filtered video (ignoring immobile AuNPs). The color scale depicts the average anomalous exponent $\alpha$ obtained from the particle trajectories (described in text). Experimental and theoretical dark-field spectra of (e) diffusive and (f) confined AuNPs at two different points in their trajectories vs gap sizes.

flat gold surface and 100-nm-diameter gold nanoparticles (AuNPs) (Figure 1a,b), similar to previous reports. ${ }^{27}$ The lipid molecules are assembled in a home-built flow-cell system, which keeps the samples hydrated. The lower leaflet of the HBL consists of octadecanethiol (ODT) assembled onto the template-stripped gold substrate. The upper leaflet is formed by the incubation of lipid vesicles containing POPC and a small amount of DOTAP (chemical structures in Figure 1c).

The charge oscillations in each AuNP couple with image charges within the polarizable $\mathrm{Au}$ mirror underneath. This enhances the electromagnetic field intensity in the gap by four orders of magnitude $\left(E^{2} / E_{0}^{2}\right)$ in an area of lipid molecules 30 $\times 30 \mathrm{~nm}^{2}$ (Figure 1d), ${ }^{28,29}$ allowing direct optical access for PL and SERS. The wavelength of light resonantly trapped in the plasmonic gap is very sensitive to the separation of the nanoparticle from the metal surface, as seen from scattering spectra calculated with finite-difference time-domain (FDTD) simulations for various gap sizes (Figure 1e) and experimentally confirmed using dark-field spectroscopy. ${ }^{27,30-32}$

Dark-field videos of this self-assembled system at room temperature (Figure 2) show three distinct types of events which help unravel the nanoscopic assembly and properties of the HBL: (i) 2D freely diffusing NPs, (ii) NPs trapped in nanoscopic wells, and (iii) blinking NPs. These events are 

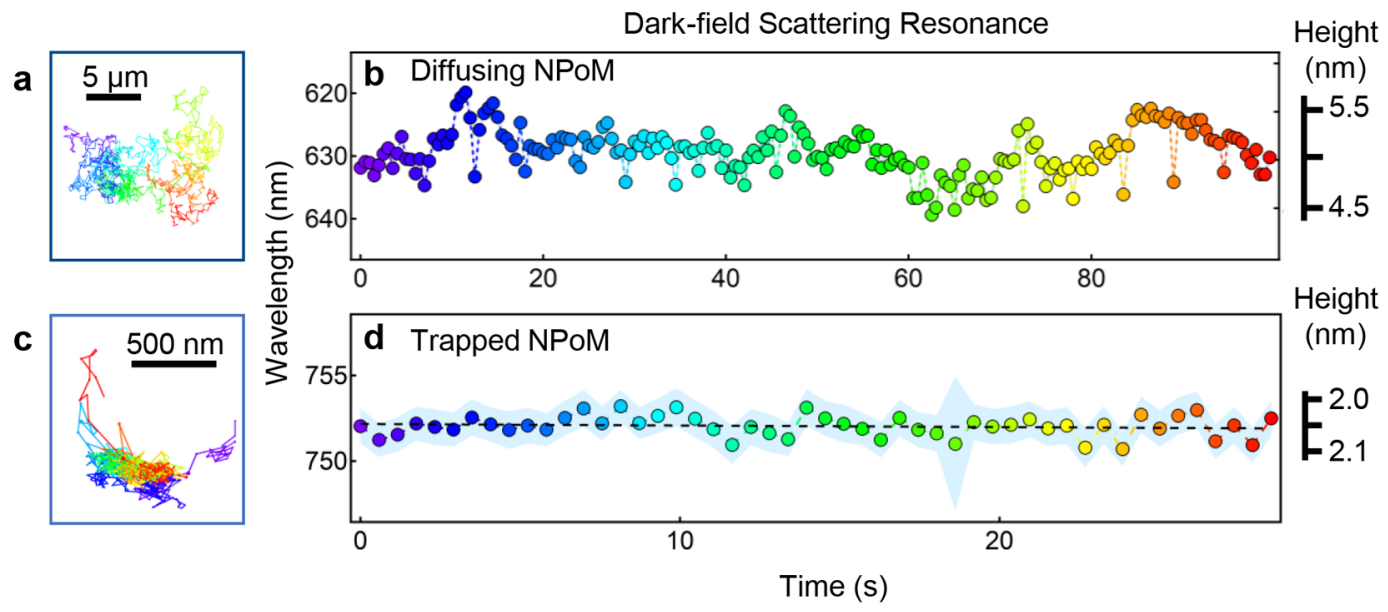

Figure 3. Tracking $z$ diffusion of the AuNPs on the lipid bilayer. In-plane $(x, y)$ particle tracks (left) and dark-field resonant peak position as a function of time (right) for ( $\mathrm{a}, \mathrm{b})$ mobile AuNPs and ( $\mathrm{c}, \mathrm{d})$ confined AuNPs. Peak positions are converted to $z$ separations using a calibrated model (see the text). Mobile AuNPs show height variations of $\sim 0.5 \mathrm{~nm}$, much larger than variations for the trapped particle.

shown schematically in Figure 2a,b, with each corresponding to a different local HBL environment around the corresponding NPoMs. The detected trajectories of NPs can be seen in Figure $2 c$, and a histogram of diffusivities from these trajectories can be seen in Figure $2 \mathrm{~d}$. The color scale here gives an indication of the average anomalous exponent (discussed in detail below) for each histogram bin. A large fraction of nanoparticles prefer to attach around defects in these bilayers and thus appear to be immobile. Au nanoparticles landing on a completely intact bilayer, however, result in freely diffusing NPs by virtue of the fluidic nature of the upper HBL leaflet. The presence of the lipid layer is verified using force-distance measurements with AFM, which give the characteristic "punch through" of the lipid layer. (See AFM images in Supporting Information Figure S8.) AFM also shows defects in the template-stripped $\mathrm{Au}$ surface and the preferential adsorption of AuNPs within these defects; however, these give weakly scattering NPs which are not captured in dark-field images.

These observations are complemented by the detailed characterization of the dynamics using time-dependent darkfield spectra collected on individual NPoMs in the diffusing and trapped states. For NPoMs that are diffusing, the dark-field resonance is always observed to be at $650 \mathrm{~nm}$ (Figure 2e). This corresponds to gap sizes of $z=5$ to $6 \mathrm{~nm}$, which agree with the expected height of the HBL. However, for trapped NPoMs the resonance is further red-shifted to $750 \mathrm{~nm}$ (Figure 2f), which corresponds to a gap size of $2 \mathrm{~nm}$, the approximate thickness of the ODT monolayer.

To understand the diffusion of NPs on an HBL, the diffusion of many individual NPoMs are tracked. Many of the NPoMs are found to diffuse along micrometer lengths with a typical diffusivity of $1.5 \mu \mathrm{m}^{2} \mathrm{~s}^{-1}$ (Figure 2d), similar to previous measurements using fluorescence imaging. ${ }^{33}$ Correlating the dark-field videos (Supporting Information) with tracks from the 2D diffusion of individual NPoMs, we collect the spectroscopic signatures while nanoparticles diffuse laterally across the HBL (Figure 3a). For an improved signal-to-noise ratio, the spectra are integrated over $500 \mathrm{~ms}$ compared to images acquired at $50 \mathrm{~ms}$. The tracked dark-field resonance of a diffusing NPoM over time reveals the $z$ motion of the AuNP height on the HBL (Figure 3b), and time-resolved dark-field spectra of more diffusing NPoMs are shown in the Supporting
Information (Figure S4). Changes in the resonance wavelength reveal out-of-plane motion of up to $1 \mathrm{~nm}$, subnanoscopic motion which has been hypothesized ${ }^{34,35}$ but never before measured.

The dark-field videos (Figure 2c) show that the diffusing NPs are excluded from the approaching trapped NPoMs closer than $3 \mu \mathrm{m}$, more than $30 \times$ larger than the NP size, repelling the diffusing NPs. Single-particle tracking of all NPs in the recorded video (Figure $\mathrm{S} 2 \mathrm{a}-\mathrm{c}$, images with all tracks) shows this clearly from histograms of the spacing between nearestneighbors (Figure S3). Our observation is unexpected since AFM confirms that the lipid layer is intact right up to the defect without displaying any topographical variation (Figure S8). This implies that the "exclusion zone" is likely electrostatic in nature and may be related to the recruitment of charged DOTAP lipids by the NP, creating a repulsive "raft" in the lipid layer (a form of quasi-2D charged double layer). The NP diffusion rate is not significantly affected by the DOTAP concentration (Figure S2d-f). However, the exclusion area strongly depends on [DOTAP] since the number of DOTAP molecules needed to stabilize the NP on the HBL remains fixed while the total number of available DOTAP molecules changes (Figure S3d,e). The boundary of the exclusion zone is sharp for larger DOTAP concentrations but more diffuse for smaller concentrations. An additional observation is that more NPs diffuse freely in $2 \mathrm{D}$ on the membrane surface at higher [DOTAP], although the mean diffusivity of the diffusing NPs is not affected significantly. We also note that for $0 \%$ DOTAP, only immobile NPs are observed.

Tracking the movement of the trapped NPoMs shows spatial diffusion confined to wells of $<1 \mu \mathrm{m}$ diameter with diffusivity of $<0.1 \mu \mathrm{m}^{2} \mathrm{~s}^{-1}$ (Figure 3c), while the dark-field resonance remains constant (Figure $3 \mathrm{~d}$ ). For hindered or confined diffusion, the mean-squared displacement of the particle is no longer expected to scale linearly with observation time and is said to be subdiffusive, ${ }^{36,37}$ empirically scaling with time to a power of $\alpha$ as $\left\langle\Delta r^{2}\right\rangle=4 \Gamma \Delta t^{\alpha}$. The diffusivity is replaced with transport coefficient $\Gamma$, and $\alpha$ gives a measure of the degree to which the diffusion is anomalous, with 1 being normal diffusion and 0 being fully confined (often referred to as compartmentalized). Many AuNPs were tested for their subdiffusive properties, and it was found that for faster-moving 
a

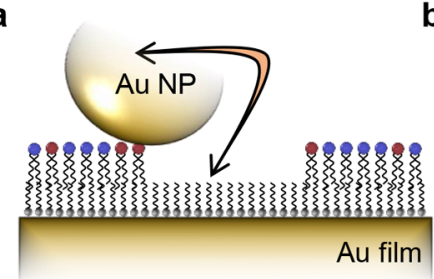

b

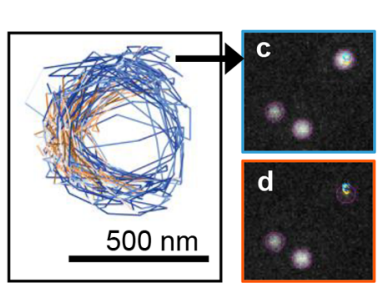

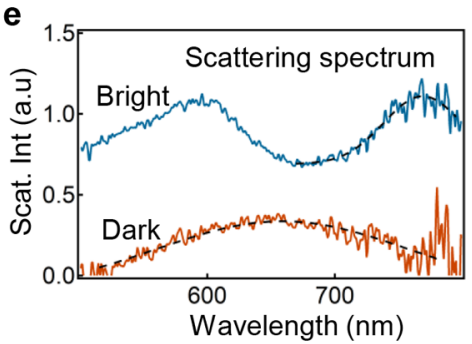

f

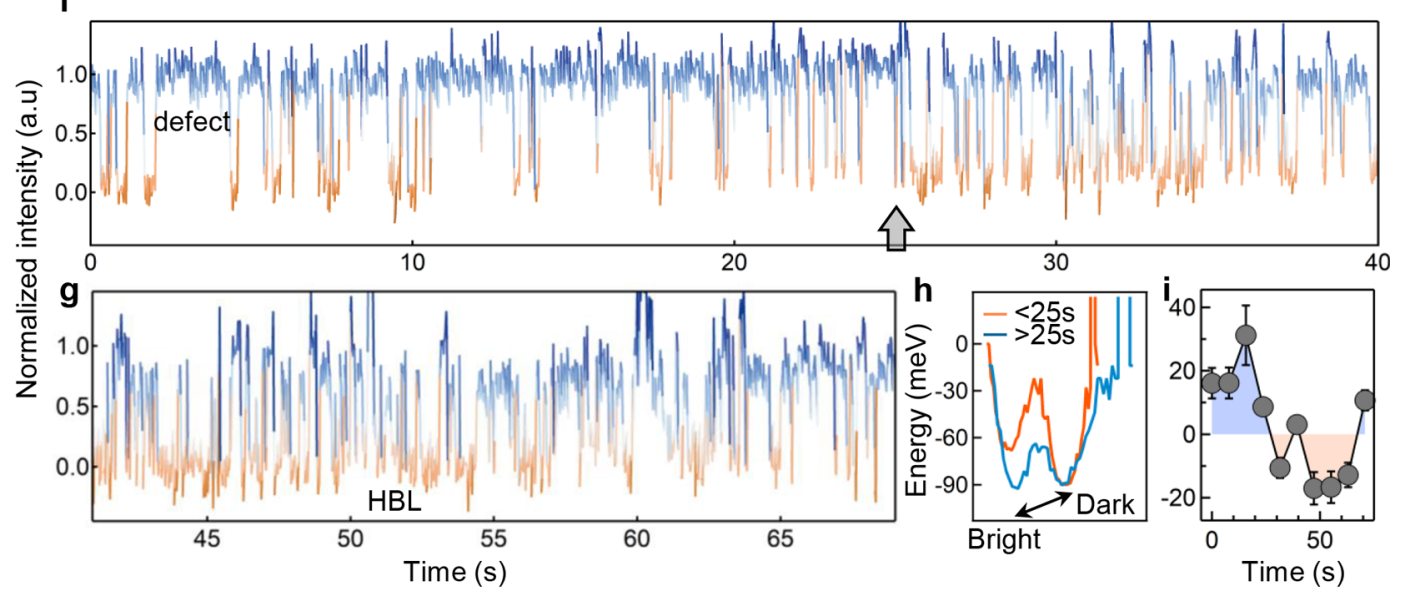

Figure 4. Dynamics of bistable AuNP on HBL. (a) Schematic motion of AuNPs that exhibits bistable confinement. (b) Particle track for one such confined AuNP and (c, d) dark-field images showing this particle (upper right), classified into two distinct intensity states (red and blue). (e) Darkfield spectra for these two states, showing different intensity and resonant peak positions. (f, $g$ ) Time trace of dark-field intensity for this particle showing binary switching behavior. After $25 \mathrm{~s}$, the particle switches to being more likely to be found in the dark state. (h) Energy potential of bright and dark states estimated from intensity fluctuations before and after $25 \mathrm{~s}$. (i) Equilibrium energies extracted from the bright/dark transition rates.

particles diffusion is normal $(\alpha \approx 1)$; however, for the confined and slower-moving particles, diffusion was found to be anomalous. For these subdiffusive particles, even at the shortest time scales the diffusion is anomalous (typically $\alpha<$ 0.5 ); hence, if the normal diffusion coefficient is taken to be 1.5 $\mu \mathrm{m}^{2} \mathrm{~s}^{-1}$, then the compartment sizes must typically be smaller than $\sim 1 \mu \mathrm{m}$. (The extraction of anomalous exponents, characteristic times, and length scales is detailed in Supporting Information, with examples in Figure S9.) This agrees with our other observations.

In rare instances, we observe nanoparticles in a bistable state, trapped at the boundary between bilayers and defects, resulting in the NP fluctuating between being in and out of the defects (schematic in Figure 4a). Such NPs are identified as blinking nanoparticles in the dark-field video (frames in Figure 4c,d; full video in Supporting Information). Dark-field spectra of the nanoparticle in the dark state show a resonance at $650 \mathrm{~nm}$ corresponding to the larger gap size of the full HBL. However, the resonance at $750 \mathrm{~nm}$ in the bright state corresponds to the smaller gap within defects formed of the bare ODT monolayer. Tracking this intensity over $30 \mathrm{~s}$ reveals the local 3D morphology of the HBL (Figure 4b). The assembly of lipids around the HBL edge is not static; there is a clear change in the statistics of flipping dynamics after $25 \mathrm{~s}$. Initially, the nanoparticle is more stable on the HBL, whereas after $25 \mathrm{~s}$ the nanoparticle appears to spend more time in the defect (Figure $4 \mathrm{f}, \mathrm{g}$ ), indicating the dynamic reassembly of lipids in the raft at the base of the nanoparticle. Comparing the intensity histogram before and after $t=25 \mathrm{~s}$ indicates that the probability of the NP being on the bilayer (dark state) has not changed but the surface energy on the defect (bright state) is modified as the NP explores the surface.

To further quantify this flipping rate, intensity traces are classified into dark/bright states (Supporting Information, Figure S7a) and the rate of flipping the HBL on and off is estimated. This allows us to extract the trapping energy $U(r)=$ $-k_{\mathrm{B}} T \ln \left(k_{\mathrm{on}} / k_{\text {off }}\right)$, with $k_{\mathrm{B}}$ being the Boltzmann constant and $T$ being the bath temperature. From these estimated energies (Figure 4i and Figure S7b), the NP is more stable in the defect during the first $20 \mathrm{~s}$, and later the equilibrium shifts to the HBL. The estimated equilibrium energies are $<20 \mathrm{meV}$, allowing the thermal bath to drive these modifications. The tethering of the NP to this edge could be due to the interplay of interactions with cationic DOTAP lipids and with the defect, which would explain why the NP never enters the central region of the defect. We note that the local heating caused by the enhanced optical absorption of AuNPs has negligible effects and does not alter the diffusion of NPoMs or the local refractive index. The temperature of AuNPs measured via the anti-Stokes Raman scattering matches the ambient room temperature within the experimental error (Figure S10). Additionally, the diffusivities of mobile NPoMs remain unchanged for different dark-field white-light intensities (Figure S11). This shows that the optical absorption of NPoMs has negligible effects on the diffusion dynamics measured here.

To further explore this diffusion and reorganization of lipids around trapped NPoMs, a small fraction ( $0.5 \%$ molar ratio) of lipid molecules tagged with fluorescent dye Texas Red (TxR, Figure 5a) were introduced. The TxR-doped HBL is excited with a $532 \mathrm{~nm}$ laser where TxR strongly absorbs (Supporting 

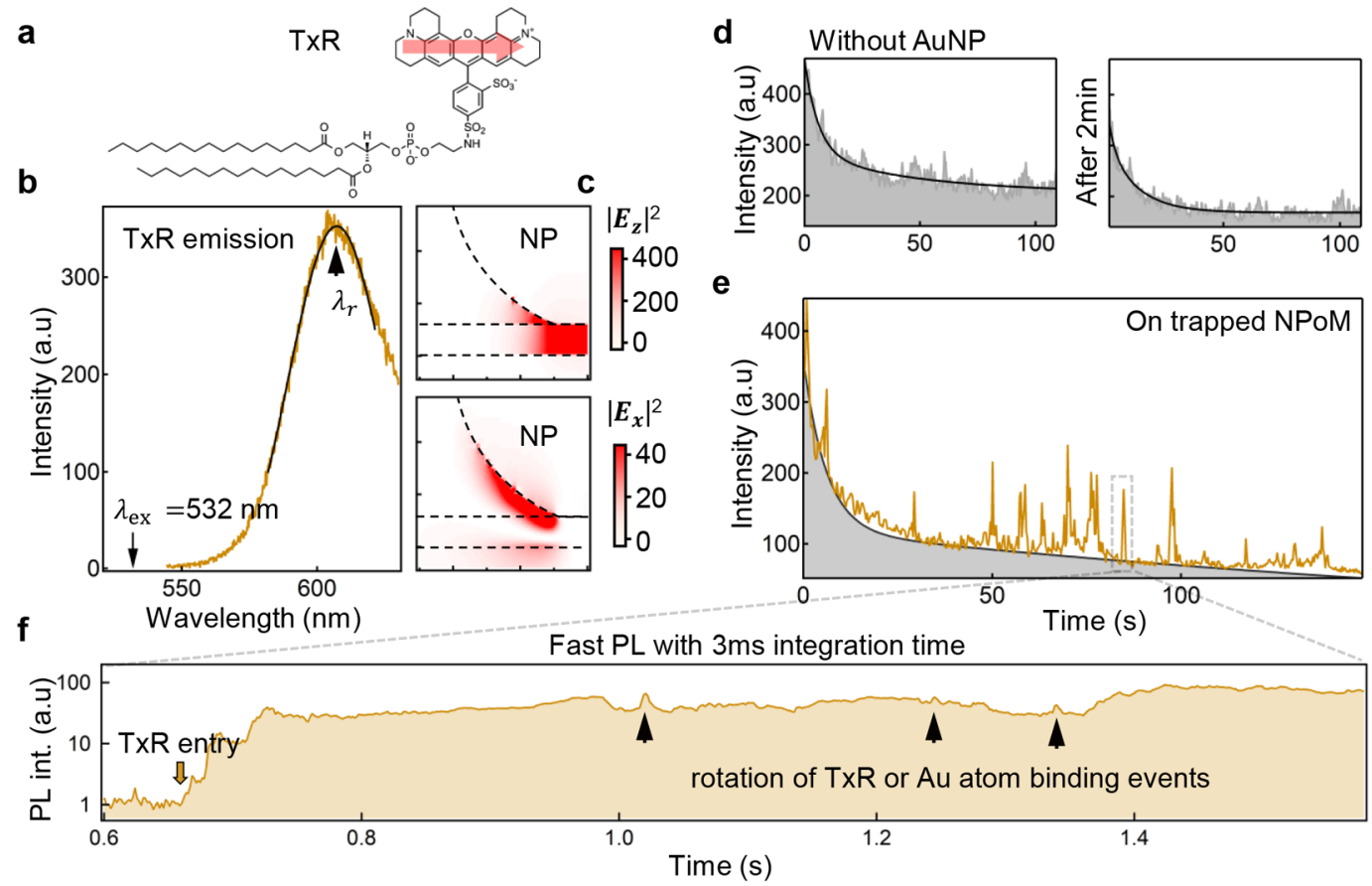

Figure 5. Dye dynamics in HBL near AuNP. (a) Chemical structure of the Texas Red (TxR)-labeled lipid molecule used to dope the HBL at a $0.5 \%$ molar ratio. The transition dipole orientation of TxR is indicated with the red arrow. (b) Fluorescence emission from TxR in HBL inside NPoM gaps, excited by a $532 \mathrm{~nm}$ laser. (c) Near-field intensity enhancement around NPoM calculated at $\lambda_{\mathrm{r}}$ for $E_{z}$ (top) and $E_{x}$ (bottom) field components. (d) Monitored intensity of TxR away from NPoM, exhibiting bleaching dynamics fitted with a double exponential (black curve). Intensity from the same area partially recovers when the laser is switched off for $2 \mathrm{~min}$ and then on again (right). The integration time is $50 \mathrm{~ms}$. (e) Similar time-series experiment on NPoM showing short bursts of fluorescence along with slow bleaching, indicating the diffusion of single TxR molecules in/out of the NPoM gap. (f) Fluorescence intensity trace acquired at fast scan rates for an integration time of 3 ms, capturing the dynamics of a single TxR lipid molecule entering the NPoM cavity and its interaction with the metal surface.

Information, Figure S6), and emission from the same diffraction-limited spot is collected by the same objective, filtered from the excitation light, and focused into a spectrometer. Fluorescence fluctuations arising from the diffusion of $\mathrm{TxR}$ in the bilayers are recorded for at least 100 s. If the HBL is irradiated away from any NPoM, then weak emission from $\mathrm{TxR}$ is observed and the intensity bleaches over time (Figure 5d). Partial recovery of the photobleached intensity is observed over the same area after $2 \mathrm{~min}$, confirming the diffusive nature of the upper leaflet of the HBL even after labeling with TxR.

Fluorescence emission from the NPoM exhibits completely different behavior (Figure 5e), with strong bursts of fluorescence superimposed on the PL from surrounding $\mathrm{HBL}$ regions, which slowly bleaches. These intense bursts represent the diffusion of single $\mathrm{TxR}$ molecules into the plasmonic nanogaps. The light is strongly confined inside the NPoM gaps, and the emission is therefore resonantly enhanced by the Purcell factor, which exceeds 1000 for a molecule with vertical dipole orientation placed in the center of the nanogap, compared to molecules in free space. ${ }^{29}$ The change in PL intensity is strongly correlated with changes in the peak of emission wavelength and the width of the PL spectra (Figure S6). As TxR molecules diffuse within the upper leaflet of the HBL around the edge of the defects, they emit strong fluorescence while entering the enhanced optical field near the NPoM. Within the diffraction-limited focal area of $\sim 1 \mu \mathrm{m}^{2}$, the number of TxR molecules expected is $\sim 1.5 \times 10^{4}$. Within the NPoM cavity mode area $\left(\sim 125 \mathrm{~nm}^{2}\right)$ there would be around 1 TxR molecule $\left(1\right.$ TxRed per $\left.130 \mathrm{~nm}^{2}\right)$. This factor of $\sim 10^{4}$ between the two is approximately the same as the enhancement factor, explaining why the emission spikes are similar in magnitude to that of the initial background fluorescence before photobleaching.

The intensity of emission is strongly influenced by the orientation of the TxR molecule in the optical fields around the NPoM. Since the TxR is covalently bound to the lipid headgroup, the transition dipole aligns mostly in plane $\left(E_{x, y}\right)$ with the weaker out-of-plane component $\left(E_{z}\right)$ arising only from the bending and flexing of lipids. The NPoM structure, however, supports stronger $E_{z}$ fields (Figure 5c) which can potentially enhance TxR emission by 500 -fold. Since these highest fields are confined inside the narrow gaps, it is not clear if they are fully accessible to the TxR, but the fluctuations within each "burst" appear to correspond to the flexing motion of the lipids. In contrast, the $E_{x}$ fields around the edge of the NPoM gap are easily accessed by the diffusing TxR, and this results in the strong bursts of fluorescence observed. For a 100 $\mathrm{nm}$ AuNP, the typical time for a freely diffusing lipid (or TxR) molecule to traverse the nanoparticle is $\sim 2.5 \mathrm{~ms}$, and considerably less time is needed to traverse the plasmonic cavity. To resolve this, we record fast PL with $3 \mathrm{~ms}$ resolution, which shows the slow diffusion of single TxR lipids into the cavity (Figure 5f; note the $\log (\mathrm{PL})$ scale). As TxR enters the cavity, the overall PL intensity increases by an average of 100fold (so the PL of a single TxR is enhanced by $\sim 10^{6}$ ), but the PL intensity still exhibits strong fluctuations associated with the rotational diffusion of the TxR. The recruitment of DOTAP molecules in the lipid layer, creating a raft under the 
AuNP, is likely to cause a significant reduction in TxR diffusion rates in and around the cavity, as we observe.

The HBLs are supported on a solid surface and consist of a single mobile layer of lipid molecules (the other layer being the immobile thiol SAM). This is likely to affect the mobility of the lipid layer and of any components embedded within the membrane (typical lipid diffusivity in a supported bilayer is $\sim 3$ $\mu \mathrm{m}^{2} \mathrm{~s}^{-1}$ but considerably higher in unsupported membranes such as in giant unilamellar vesicles ${ }^{38}$ ). Viscous drag will affect the mobility of the upper lipid layer, and surface interactions make it challenging to introduce full transmembrane proteins into this system. We thus anticipate further development to include "unsupported" lipid membranes with AuNP attachment on both sides, removing the complications of substrate interactions. The HBL model does, however, still capture many of the properties of a cell membrane, including, most importantly, the lateral fluidity of the lipid layer.

This work provides insight into the nanoscopic organization of lipid bilayers and their defects around gold nanoparticles. The corresponding motion of these AuNPs together with their spectroscopic information provides dynamic topographical information. We observe instances where NPs diffuse as freely as lipid molecules in the membrane while being electrostatically attached to the DOTAP molecules present. We also see cases where NPs sit inside defects in the bilayer and reorganize lipids around them, exhibiting confined diffusion. The latter case shows significant activity in dark-field spectra with the resonance peak shifts tracking fluctuations in $z$ height. Most importantly, we note that the NPs do not appear to perturb the lipid membrane structure other than the possible recruitment of charged components in the vicinity of pre-existing defects. From this, we conclude that the HBL system used with the NPoM geometry is well suited for studying the out-of-plane motion of lipids and diffusion associated with the membrane. Alternative models may be required if large complex moieties that span the membrane, such as proteins, are to be studied.

\section{EXPERIMENTAL SECTION}

Template-Stripped Gold. A silicon wafer is cleaned using a $10 \%$ Decon 90 solution and rinsed twice with water, ethanol, and isopropanol. A 100-nm-thick layer of gold is evaporated on the wafer at $1 \AA \mathrm{s}^{-1}$ using a Kurt J. Lesker E-beam evaporator. The $\mathrm{Au}$-coated silicon wafer was heated to $60{ }^{\circ} \mathrm{C}$, and smaller pieces of silicon wafer were glued to the surface using an EpoTek 377 epoxy glue, which was cured at $150{ }^{\circ} \mathrm{C}$ for $18 \mathrm{~h}$. The wafer was then allowed to cool gradually to room temperature, and individual pieces of silicon were peeled off, exposing a flat, clean gold surface, prior to functionalization with octadecanethiol.

Hybrid Lipid Bilayer Preparation. Template-stripped gold was incubated overnight at room temperature in ethanol containing $5 \mathrm{mM}$ octadecanethiol (ODT, Sigma), forming a selfassembled monolayer (SAM). After monolayer deposition, the substrates were rinsed thoroughly with ethanol, dried under a stream of nitrogen, and used immediately. One milligram of dried palmitoyl-oleoylphosphatidylcholine (Avanti Polar Lipids, Alabaster, AL) was resuspended by vortex mixing in $1 \mathrm{~mL}$ of phosphate-buffered saline (PBS) solution (Sigma) and extruded 21 times through a $50 \mathrm{~nm}$ track-etch membrane (Whatman) using a miniextruder kit (Avanti Polar Lipids). The resulting suspension became clear, indicating the formation of small unilamellar vesicles, which were stored in the refrigerator and used within a few days. When ready for use, the vesicle suspension was diluted in PBS to a concentration of $0.5 \mathrm{mg} / \mathrm{mL}$, injected into a custom-built flow cell which contained a piece of template-stripped gold with an ODT SAM, and incubated for $60 \mathrm{~min}$. The sample was then rinsed with Milli- $Q$ water and then more PBS.

Dark-Field Microscopy. The final step in preparing the sample for imaging was to inject $100 \mathrm{~nm}$ gold nanoparticles into the flow cell. These were incubated for approximately $1 \mathrm{~min}$ before the excess was carefully rinsed away with PBS. The flow cell was then placed on the microscope stage under a dark-field $100 \times(0.8 \mathrm{NA})$ objective for imaging (optical setup, Figure S1 in the Supporting Information). The dark-field video was recorded in monochromatic mode with $50 \mathrm{~ms}$ for each frame and with a refresh time of $70 \mathrm{~ms}$ between frames. The darkfield spectra were recorded over a wide area of $5 \times 5 \mu \mathrm{m}^{2}$ at a rate of $2 \mathrm{~Hz}$ using a QE65000 Ocean Optics spectrometer while being illuminated with a halogen lamp.

Single-Particle Tracking. Videos of diffusing AuNPs were processed with FIJI software, and the AuNPs were identified and tracked using the TrackMate single particle tracking plugin. ${ }^{39}$ This yielded a collection of particle tracks which represented the motion of each of the AuNPs in the video together with their intensity values at each point in time. This information was subsequently used to obtain diffusion information including diffusivity and the anomalous exponent to help identify whether the AuNPs were undergoing free or hindered diffusion.

Atomic Force Microscopy. Atomic force microscopy (AFM) measurements were performed under fluid conditions at room temperature in tapping mode using a Dimension FastScan Bio with Fast Scan D probes (Bruker), short cantilevers with a spring constant of $\sim 0.25 \mathrm{~N} \mathrm{~m}^{-1}$, and a resonance frequency of $110 \mathrm{kHz}$. The force applied by the tip to the sample was minimized by maximizing the set point while maintaining tracking of the surface.

\section{ASSOCIATED CONTENT}

\section{Supporting Information}

The Supporting Information is available free of charge at https://pubs.acs.org/doi/10.1021/acs.jpclett.0c00182. Source data can be found at https://doi.org/10.17863/CAM.50763

Experimental setup, effect of DOTAP concentration on NPoM diffusivities, out-of-plane lipid diffusion, comparing spectral wandering of different types of NPoM, time resolved fluorescence from Texas Red-labeled lipid, edge-flipping dynamics, atomic force microscopy, and anomalous diffusion analysis (PDF)

Dark-field video showing the diffusion of AuNPs on the HBL (AVI)

\section{AUTHOR INFORMATION}

\section{Corresponding Authors}

Jeremy J. Baumberg - NanoPhotonics Centre, Cavendish Laboratory, Department of Physics, University of Cambridge, Cambridge CB3 OHE, United Kingdom; 이이.org/00000002-9606-9488; Email: jjb12@cam.ac.uk

Rohit Chikkaraddy - NanoPhotonics Centre, Cavendish Laboratory, Department of Physics, University of Cambridge, Cambridge CB3 OHE, United Kingdom; 이이.org/00000002-3840-4188; Email: rc621@cam.ac.uk 


\section{Authors}

Matthew R. Cheetham - NanoPhotonics Centre, Cavendish Laboratory, Department of Physics, University of Cambridge, Cambridge CB3 OHE, United Kingdom

Jack Griffiths - NanoPhotonics Centre, Cavendish Laboratory, Department of Physics, University of Cambridge, Cambridge CB3 OHE, United Kingdom

Bart de Nijs - NanoPhotonics Centre, Cavendish Laboratory, Department of Physics, University of Cambridge, Cambridge CB3 OHE, United Kingdom

George R. Heath - School of Physics and Astronomy, University of Leeds, Leeds LS2 9JT, United Kingdom; O orcid.org/00000001-6431-2191

Stephen D. Evans - School of Physics and Astronomy, University of Leeds, Leeds LS2 9JT, United Kingdom

Complete contact information is available at: https://pubs.acs.org/10.1021/acs.jpclett.0c00182

\section{Notes}

The authors declare no competing financial interest.

\section{ACKNOWLEDGMENTS}

We acknowledge support from EPSRC grants EP/G060649/1, EP/L027151/1, EP/G037221/1, and EPSRC NanoDTC. R.C. acknowledges support from Trinity College, University of Cambridge. B.d.N acknowledges support from the Leverhulme Trust and Isaac Newton Trust in the form of an Early Career Fellowship. S.D.E acknowledges funding from the EPSRC (EP/P023266/1).

\section{REFERENCES}

(1) Arroyo, J. O.; Kukura, P. Non-Fluorescent Schemes for SingleMolecule Detection, Imaging and Spectroscopy. Nat. Photonics 2016, 10, 11-17.

(2) Limaj, O.; Etezadi, D.; Wittenberg, N. J.; Rodrigo, D.; Yoo, D.; Oh, S.-H.; Altug, H. Infrared Plasmonic Biosensor for Real-Time and Label-Free Monitoring of Lipid Membranes. Nano Lett. 2016, 16, $1502-1508$

(3) Guo, Y.; Terazzi, E.; Seemann, R.; Fleury, J. B.; Baulin, V. A. Direct Proof of Spontaneous Translocation of Lipid-Covered Hydrophobic Nanoparticles through a Phospholipid Bilayer. Sci. Adv. 2016, 2, No. e1600261.

(4) Li, Y.; Lin, T.; Luo, Y.; Liu, Q.; Xiao, W.; Guo, W.; Lac, D.; Zhang, H.; Feng, C.; Wachsmann-Hogiu, S.; et al. A Smart and Versatile Theranostic Nanomedicine Platform Based on Nanoporphyrin. Nat. Commun. 2014, 5, 4712.

(5) Lin, J.; Zhang, H.; Chen, Z.; Zheng, Y. Penetration of Lipid Membranes by Gold Nanoparticles: Insights into Cellular Uptake, Cytotoxicity, and Their Relationship. ACS Nano 2010, 4, 5421-5429.

(6) Johnston, H. J.; Hutchison, G.; Christensen, F. M.; Peters, S.; Hankin, S.; Stone, V. A Review of the in Vivo and in Vitro Toxicity of Silver and Gold Particulates: Particle Attributes and Biological Mechanisms Responsible for the Observed Toxicity. Crit. Rev. Toxicol. 2010, 40, 328-346.

(7) Zimmerman, J. F.; Ardoña, H. A. M.; Pyrgiotakis, G.; Dong, J.; Moudgil, B.; Demokritou, P.; Parker, K. K. Scatter Enhanced Phase Contrast Microscopy for Discriminating Mechanisms of Active Nanoparticle Transport in Living Cells. Nano Lett. 2019, 19, 793804.

(8) Lesniak, A.; Fenaroli, F.; Monopoli, M. P.; Åberg, C.; Dawson, K. A.; Salvati, A. Effects of the Presence or Absence of a Protein Corona on Silica Nanoparticle Uptake and Impact on Cells. ACS Nano 2012, 6, 5845-5857.
(9) Chithrani, B. D.; Chan, W. C. W. Elucidating the Mechanism of Cellular Uptake and Removal of Protein-Coated Gold Nanoparticles of Different Sizes and Shapes. Nano Lett. 2007, 7, 1542-1550.

(10) Singer, S. J.; Nicolson, G. L. The Fluid Mosaic Model of the Structure of Cell Membranes. Science 1972, 175, 720-731.

(11) Ritchie, K.; Iino, R.; Fujiwara, T.; Murase, K.; Kusumi, A. The Fence and Picket Structure of the Plasma Membrane of Live Cells as Revealed by Single Molecule Techniques (Review). Mol. Membr. Biol. 2003, 20, 13-18.

(12) Tarun, O. B.; Hannesschläger, C.; Pohl, P.; Roke, S. Label-Free and Charge-Sensitive Dynamic Imaging of Lipid Membrane Hydration on Millisecond Time Scales. Proc. Natl. Acad. Sci. U. S. A. 2018, 115, 4081-4086.

(13) Coker, H. L. E.; Cheetham, M. R.; Kattnig, D. R.; Wang, Y. J.; Garcia-Manyes, S.; Wallace, M. I. Controlling Anomalous Diffusion in Lipid Membranes. Biophys. J. 2019, 116, 1085-1094.

(14) Chee, S. W.; Anand, U.; Bisht, G.; Tan, S. F.; Mirsaidov, U. Direct Observations of the Rotation and Translation of Anisotropic Nanoparticles Adsorbed at a Liquid-Solid Interface. Nano Lett. 2019, 19, 2871-2878.

(15) Fu, X.; Chen, B.; Tang, J.; Hassan, M. T.; Zewail, A. H. Imaging Rotational Dynamics of Nanoparticles in Liquid by 4D Electron Microscopy. Science 2017, 355, 494-498.

(16) Regmi, R.; Winkler, P. M.; Flauraud, V.; Borgman, K. J. E.; Manzo, C.; Brugger, J.; Rigneault, H.; Wenger, J.; García-Parajo, M. F. Planar Optical Nanoantennas Resolve Cholesterol-Dependent Nanoscale Heterogeneities in the Plasma Membrane of Living Cells. Nano Lett. 2017, 17, 6295-6302.

(17) Winkler, P. M.; Regmi, R.; Flauraud, V.; Brugger, J.; Rigneault, H.; Wenger, J.; García-Parajo, M. F. Optical Antenna-Based Fluorescence Correlation Spectroscopy to Probe the Nanoscale Dynamics of Biological Membranes. J. Phys. Chem. Lett. 2018, 9, 110-119.

(18) Winkler, P. M.; Regmi, R.; Flauraud, V.; Brugger, J.; Rigneault, H.; Wenger, J.; García-Parajo, M. F. Transient Nanoscopic Phase Separation in Biological Lipid Membranes Resolved by Planar Plasmonic Antennas. ACS Nano 2017, 11, 7241-7250.

(19) Taylor, R. W.; Mahmoodabadi, R. G.; Rauschenberger, V.; Giessl, A.; Schambony, A.; Sandoghdar, V. Interferometric Scattering Microscopy Reveals Microsecond Nanoscopic Protein Motion on a Live Cell Membrane. Nat. Photonics 2019, 13, 480-487.

(20) Taylor, R. W.; Sandoghdar, V. Interferometric Scattering Microscopy: Seeing Single Nanoparticles and Molecules via Rayleigh Scattering. Nano Lett. 2019, 19, 4827-4835.

(21) de Wit, G.; Danial, J. S. H.; Kukura, P.; Wallace, M. I. Dynamic Label-Free Imaging of Lipid Nanodomains. Proc. Natl. Acad. Sci. U. S. A. 2015, 112, 12299-12303.

(22) de Wit, G.; Albrecht, D.; Ewers, H.; Kukura, P. Revealing Compartmentalized Diffusion in Living Cells with Interferometric Scattering Microscopy. Biophys. J. 2018, 114, 2945-2950.

(23) Brolo, A. G. Plasmonics for Future Biosensors. Nat. Photonics 2012, 6, 709-713.

(24) Baumberg, J. J.; Aizpurua, J.; Mikkelsen, M. H.; Smith, D. R Extreme Nanophotonics from Ultrathin Metallic Gaps. Nat. Mater. 2019, 18, 668-678.

(25) Baaske, M. D.; Foreman, M. R.; Vollmer, F. Single-Molecule Nucleic Acid Interactions Monitored on a Label-Free Microcavity Biosensor Platform. Nat. Nanotechnol. 2014, 9, 933-939.

(26) Lum, W.; Bruzas, I.; Gorunmez, Z.; Unser, S.; Beck, T.; Sagle, L. Novel Liposome-Based Surface-Enhanced Raman Spectroscopy (SERS) Substrate. J. Phys. Chem. Lett. 2017, 8, 2639-2646.

(27) Taylor, R. W.; Benz, F.; Sigle, D. O.; Bowman, R. W.; Bao, P.; Roth, J. S.; Heath, G. R.; Evans, S. D.; Baumberg, J. J. Watching Individual Molecules Flex within Lipid Membranes Using SERS. Sci. Rep. 2015, 4, 5940.

(28) Kongsuwan, N.; Demetriadou, A.; Chikkaraddy, R.; Benz, F.; Turek, V. A.; Keyser, U. F.; Baumberg, J. J.; Hess, O. Suppressed Quenching and Strong-Coupling of Purcell-Enhanced Single-Mole- 
cule Emission in Plasmonic Nanocavities. ACS Photonics 2018, 5, $186-191$.

(29) Chikkaraddy, R.; Turek, V. A.; Kongsuwan, N.; Benz, F.; Carnegie, C.; van de Goor, T.; de Nijs, B.; Demetriadou, A.; Hess, O.; Keyser, U. F.; et al. Mapping Nanoscale Hotspots with SingleMolecule Emitters Assembled into Plasmonic Nanocavities Using DNA Origami. Nano Lett. 2018, 18, 405-411.

(30) Chikkaraddy, R.; Zheng, X.; Benz, F.; Brooks, L. J.; de Nijs, B.; Carnegie, C.; Kleemann, M.-E.; Mertens, J.; Bowman, R. W.; Vandenbosch, G. A. E.; et al. How Ultranarrow Gap Symmetries Control Plasmonic Nanocavity Modes: From Cubes to Spheres in the Nanoparticle-on-Mirror. ACS Photonics 2017, 4, 469-475.

(31) de Nijs, B.; Bowman, R. W.; Herrmann, L. O.; Benz, F.; Barrow, S. J.; Mertens, J.; Sigle, D. O.; Chikkaraddy, R.; Eiden, A.; Ferrari, A.; Scherman, O. A.; Baumberg, J. J.; et al. Unfolding the Contents of Sub-nm Plasmonic Gaps Using Normalising Plasmon Resonance Spectroscopy. Faraday Discuss. 2015, 178, 185-193.

(32) Kleemann, M.-E.; Mertens, J.; Zheng, X.; Cormier, S.; Turek, V.; Benz, F.; Chikkaraddy, R.; Deacon, W.; Lombardi, A.; Moshchalkov, V. V.; et al. Revealing Nanostructures through Plasmon Polarimetry. ACS Nano 2017, 11, 850-855.

(33) Fujiwara, T.; Ritchie, K.; Murakoshi, H.; Jacobson, K.; Kusumi, A. Phospholipids Undergo Hop Diffusion in Compartmentalized Cell Membrane. J. Cell Biol. 2002, 157, 1071-1082.

(34) Lee, Y. K.; Kim, S.; Oh, J.-W.; Nam, J.-M. Massively Parallel and Highly Quantitative Single-Particle Analysis on Interactions between Nanoparticles on Supported Lipid Bilayer. J. Am. Chem. Soc. 2014, 136, 4081-4088.

(35) Liao, Y.-H.; Lin, C.-H.; Cheng, C.-Y.; Wong, W. C.; Juo, J.-Y.; Hsieh, C.-L. Monovalent and Oriented Labeling of Gold Nanoprobes for the High-Resolution Tracking of a Single-Membrane Molecule. ACS Nano 2019, 13, 10918-10928.

(36) Saxton, M. J. A Biological Interpretation of Transient Anomalous Subdiffusion. II. Reaction Kinetics. Biophys. J. 2008, 94, $760-771$.

(37) Murase, K.; Fujiwara, T.; Umemura, Y.; Suzuki, K.; Iino, R.; Yamashita, H.; Saito, M.; Murakoshi, H.; Ritchie, K.; Kusumi, A. Ultrafine Membrane Compartments for Molecular Diffusion as Revealed by Single Molecule Techniques. Biophys. J. 2004, 86, 4075-4093.

(38) Przybylo, M.; Sýkora, J.; Humpolícková, J.; Benda, A.; Zan, A.; Hof, M. Lipid Diffusion in Giant Unilamellar Vesicles Is More than 2 Times Faster than in Supported Phospholipid Bilayers under Identical Conditions. Langmuir 2006, 22, 9096-9099.

(39) Tinevez, J.-Y.; Perry, N.; Schindelin, J.; Hoopes, G. M.; Reynolds, G. D.; Laplantine, E.; Bednarek, S. Y.; Shorte, S. L.; Eliceiri, K. W. TrackMate: An Open and Extensible Platform for SingleParticle Tracking. Methods 2017, 115, 80-90. 